\title{
Flexible Self-Resonant Detector Coil for Magnetic Resonance Imaging of Carbon-13
}

Zhurbenko, Vitaliy; Sanchez-Heredia, Juan D.; Wang, Wenjun; Ardenkjær-Larsen, Jan H.

\section{Published in:}

Proceedings of 2020 European Microwave Conference

Link to article, DOI:

10.23919/EuMC48046.2021.9338182

Publication date:

2021

Document Version

Peer reviewed version

Link back to DTU Orbit

\section{Citation (APA):}

Zhurbenko, V., Sanchez-Heredia, J. D., Wang, W., \& Ardenkjær-Larsen, J. H. (2021). Flexible Self-Resonant Detector Coil for Magnetic Resonance Imaging of Carbon-13. In Proceedings of 2020 European Microwave Conference (pp. 112-115). IEEE. https://doi.org/10.23919/EuMC48046.2021.9338182

\section{General rights}

Copyright and moral rights for the publications made accessible in the public portal are retained by the authors and/or other copyright owners and it is a condition of accessing publications that users recognise and abide by the legal requirements associated with these rights.

- Users may download and print one copy of any publication from the public portal for the purpose of private study or research.

- You may not further distribute the material or use it for any profit-making activity or commercial gain

- You may freely distribute the URL identifying the publication in the public portal 


\title{
Flexible Self-Resonant Detector Coil for Magnetic Resonance Imaging of Carbon-13
}

\author{
Vitaliy Zhurbenko, Juan D. Sánchez-Heredia, Wenjun Wang, Jan H. Ardenkjær-Larsen \\ Technical University of Denmark \\ vz@elektro.dtu.dk
}

\begin{abstract}
Magnetic Resonance is a powerful imaging modality which relies on arrays of detector coils to collect weak RF signals. Parasitic coupling between array elements degrades imaging system performance and greatly complicates array design process. In this work we show how to significantly reduce interaction between array elements by employing a modified highimpedance coil structure and combining it with a noise-matched low-input-impedance LNA.

The approach is verified experimentally by designing a two element array for Magnetic Resonance Imaging (MRI) of carbon-13. The fabricated detector demonstrates over $35 \mathrm{~dB}$ decoupling between array elements. Imaging experiments in a 3 tesla MRI scanner showed very low variation of noise correlation between channels in the array. The coils utilize flexible conductors and suitable for construction of flexible arrays conformal to different patient sizes.

The presented approach is useful in the design of antenna arrays and RF detector arrays where channel-to-channel interaction creates a significant problem.
\end{abstract}

Keywords - biomedical electronics, air coils, detectors, lownoise amplifiers, magnetic resonance imaging, mutual coupling.

\section{INTRODUCTION}

The recent trend in MRI detector development is using multichannel arrays. They offer better sensitivity and allow for parallel data acquisition, which leads to higher resolution and faster scan times compared to more traditional volume detectors. The detectors are typically constructed from loop coils which sense RF magnetic field. The coils in vicinity of each other couple magnetically, which results in detuning and detection sensitivity degradation. Magnetic coupling can be compensated by partially overlapping the coils, which works well for neighbor array elements but is not possible for next neighbor [1]. Recently, self-resonant, so called, high-impedance coils have been suggested promising better decoupling between array elements [2]. They are constructed from a coaxial cable and their resonance frequency is strictly defined by the coil loop size and dielectric properties of the cable insulator. This dependence is not always convenient, since the coil size is typically chosen based on the size of the object to image. Several methods have been suggested to tune the coil while keeping the coil size fixed in order to increase the design flexibility [3] and make the technology more suitable for a wider range of MRI systems and nuclei. Most of these methods are better suitable for increasing the operating frequency. MRI of low gyromagnetic ratio nuclei using standard clinical scanners typically requires lowering the resonance frequency of the high-impedance coil if a standard $50 \Omega$ coaxial cable is used. One method to decrease the operating frequency is to use multi-turn coils [3]. The approach leads to high quality factor coils, while impairs their mechanical flexibility.

In this work we propose a different approach to construct a high-impedance coil, which allows tuning the coil to virtually any frequency using a standard $50 \Omega$ coaxial cable while preserving a high Q-factor. This is achieved by including a lumped capacitor $C_{\mathrm{t}}$ in the gap in the outer conductor of the coaxial cable, as shown in Fig. 1.

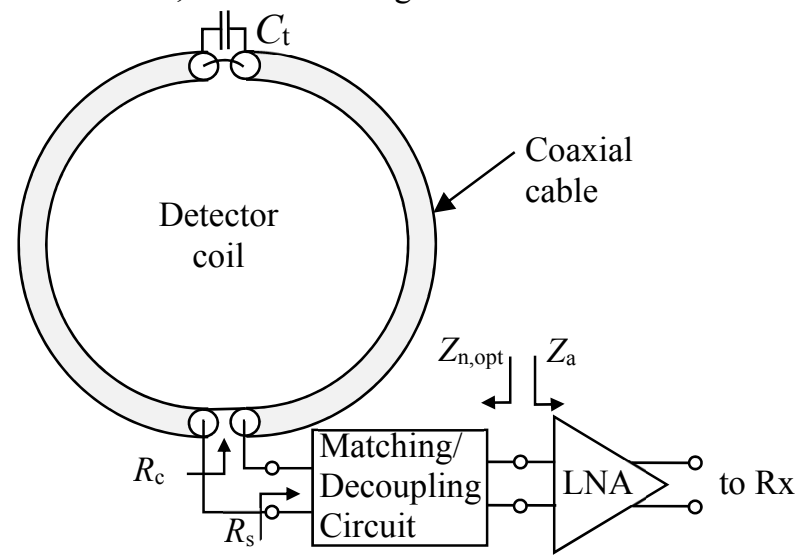

Fig. 1. A modified high-impedance detector coil in combination with preamplifier decoupling circuit.

To further increase decoupling properties of the detector coil, it is connected to a low noise amplifier (LNA) through impedance transformation network. The network transforms the output impedance of the coil, $R_{\mathrm{c}}$, to the optimal noise impedance of the LNA, $Z_{\mathrm{n}, \mathrm{opt}}$, and, at the same time, transforms the input impedance of the LNA, $Z_{\mathrm{a}}$, to low impedance at the terminals of the coil, $R_{\mathrm{s}}$, maximizing the decoupling.

\section{High-IMPEDANCE DETECTOR}

\section{A. Structure}

The high-impedance detector coil is a self-resonant structure consisting of a loop shaped coaxial cable, where the inner conductor is used as detector terminals and a gap is introduced in the outer conductor on the opposite side from the terminals of the loop. The self-inductance of the inner conductor resonates with the capacitor formed by the outer and 
inner conductors separated by the cable insulator. In a simplified form the coil can be modelled as a parallel resonator formed by this inductance and capacitance, which, at resonance frequency exhibits a high output impedance. Therefore it is often referenced in the literature as a high-impedance coil [2]. In this work, it is proposed to tune the resonance frequency of the coil using hybrid integration of lumped components in the coil structure. Several topologies are possible, which will lead to reducing the resonance frequency: including a parallel capacitor at the output terminals of the coil; inserting an inductor instead of a short circuit in the outer conductor at the side of the output terminals; inserting a capacitor in the gap as shown in Fig. 1; or a combination of all above mentioned. Fullwave electromagnetic analysis of all suggested topologies showes, that the arrangement in Fig. 1 is the most efficient of the options when implementing a coaxial cable with a typical loss level.

\section{B. Design Approach}

The design approach of this detector is straightforward. First, the diameter of the loop is chosen from imaging considerations. The response of the coaxial loop can be modelled either in the 3D EM simulator, or in a circuit simulator using two sections of a coaxial cable of the corresponding length $l(l \approx \pi r$, where $r$ is the radius of the loop). The loop coil exhibits inductive output impedance below the self-resonance frequency. The capacitance $C_{\mathrm{t}}$ can then be chosen to resonate the loop at the desired frequency.

To sustain the efficiency of the detector, the output impedance of the coil, $R_{\mathrm{c}}$, must be transformed to an optimal noise impedance of the LNA, $Z_{\mathrm{n} \text {,opt }}=R_{\mathrm{n}, \mathrm{opt}}+\mathrm{j} X_{\mathrm{n} \text {,opt. }}$ This is achieved with the matching and decoupling circuit, as can be seen in Fig. 1. To minimize the influence of the loop coil on other array elements, its output terminals can be short circuited. In practice, this is achieved by transforming input impedance of the LNA, $Z_{\mathrm{a}}=R_{\mathrm{a}}+\mathrm{j} X_{\mathrm{a}}$, to the lowest possible impedance $R_{\mathrm{s}}$. Assuming the matching and decoupling network is lossless, the level of mismatch at the coil and LNA terminals will be the same [4] and in this case can be estimated from $Z_{\mathrm{a}}$ and $Z_{\mathrm{n}, \mathrm{opt}}$ parameters of a given LNA. The achievable level of $R_{\mathrm{S}}$ can be calculated as:

where

$$
R_{s}=\frac{R_{C}\left(1-\left|\Gamma_{a}\right|\right)}{1+\left|\Gamma_{a}\right|}
$$

$$
\Gamma_{a}=\frac{R_{a}+j\left(X_{a}+X_{n, o p t}\right)-R_{n, o p t}}{R_{a}+j\left(X_{a}+X_{n, o p t}\right)+R_{n, o p t}} .
$$
$R_{\mathrm{s}}$.

The LNA can then be chosen by comparing the achievable

\section{DESIGN EXAMPLE}

A two element array for imaging of carbon-13 in a $3 \mathrm{~T}$ medical scanner was developed and tested to demonstrate the design approach. The operating frequency, $f_{0}$, is in this case 32.1 MHz.

\section{A. Coil Construction}

The $8 \mathrm{~cm}$ diameter of the coil is chosen to be the same as the diameter of a standard low-impedance reference coil for these applications [7]. A flexible $50 \Omega$ coaxial cable of $1 \mathrm{~mm}$ outer diameter is used to construct the coil. In order to resonate the coil at the desired frequency, $f_{0}$, a capacitor $C_{\mathrm{t}}=121 \mathrm{pF}$ ( $120 \mathrm{pF}$ and $1 \mathrm{pF}$ capacitors in parallel) is implemented, which was found by EM simulations and verified by measurements of the resonance frequency with a VNA. The resulting output impedance of the coil, $R_{c}$, at $f_{0}$ when placed next to the phantom is $2 \mathrm{k} \Omega$.

\section{B. Matching/Decoupling Circuit and LNA}

An off-the-shelf LNA from ElCry [5] has been used with the following parameters: $Z_{\mathrm{a}} \approx 64-\mathrm{j} 647 \Omega$ and $Z_{\mathrm{n}, \mathrm{opt}} \approx 116+\mathrm{j} 19 \Omega$. Substituting these parameters into eq. (1) leads to $R_{\mathrm{s}} \approx 36 \Omega$. This is not a very low impedance by conventional standards, but it is much lower than $R_{\mathrm{c}}$, which leads to a high level of decoupling, as it will be shown below. The matching and decoupling circuit is designed by solving a system of equations from circuit theory relating circuit elements and input/output impedances of the circuit [6]. As described above, the following conditions are imposed on the circuit: for minimum noise, the output impedance of the circuit connected to the detector coil must be equal to $Z_{\mathrm{n}, \mathrm{opt}}$; for maximum decoupling, the input impedance of the circuit connected to LNA must be equal $R_{\mathrm{s}}$. The solution leads to a three element network [6]. One element was neglected due to its low value and for the sake of simplicity of circuit realization. The resulting matching and decoupling network consists of a $6 \mathrm{pF}$ parallel capacitor and a $3 \mu \mathrm{H}$ series inductor, as shown in Fig. 2 together with achieved impedances of the LNA combined with the matching and decoupling circuit.

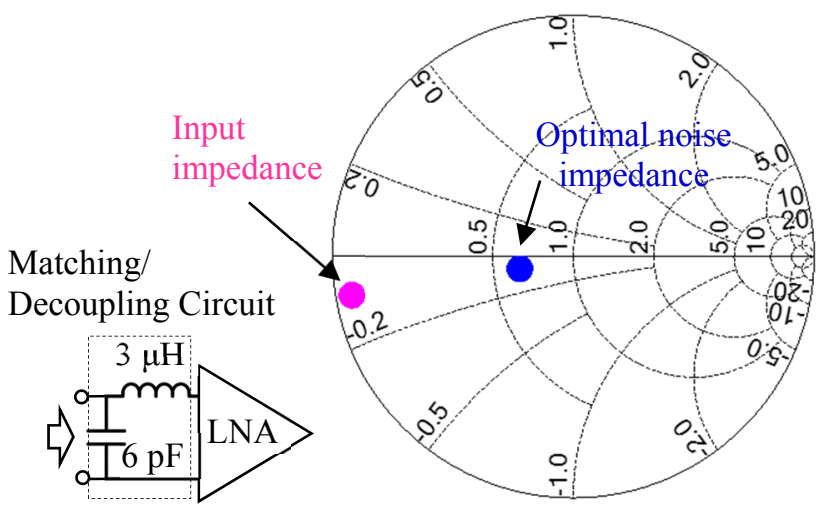

Fig. 2. Impedances at the input terminals of the matching and decoupling circuit (normalized to $R_{\mathrm{c}}$ ).

Though the resulting impedances are not ideal due to simplifications and loss in the $3 \mu \mathrm{H}$ inductor, they are relatively close to values providing optimal noise (in the center of the Chart) and decoupling conditions (closer to the short-circuit). A photograph of the fabricated prototype is shown in Fig. 3.

The decoupling properties of the detector were measured with a double-loop probe connected to a vector network analyzer [2], [6]. 


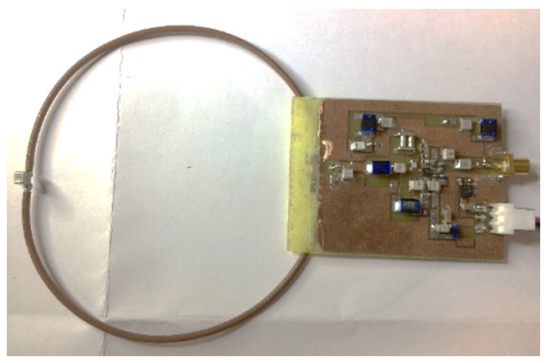

Fig. 3. A photograph of the fabricated flexible detector prototype.

Normalized amplitude of $\mathrm{S}_{21}$ for the tuned and detuned states are shown in Fig. 4.

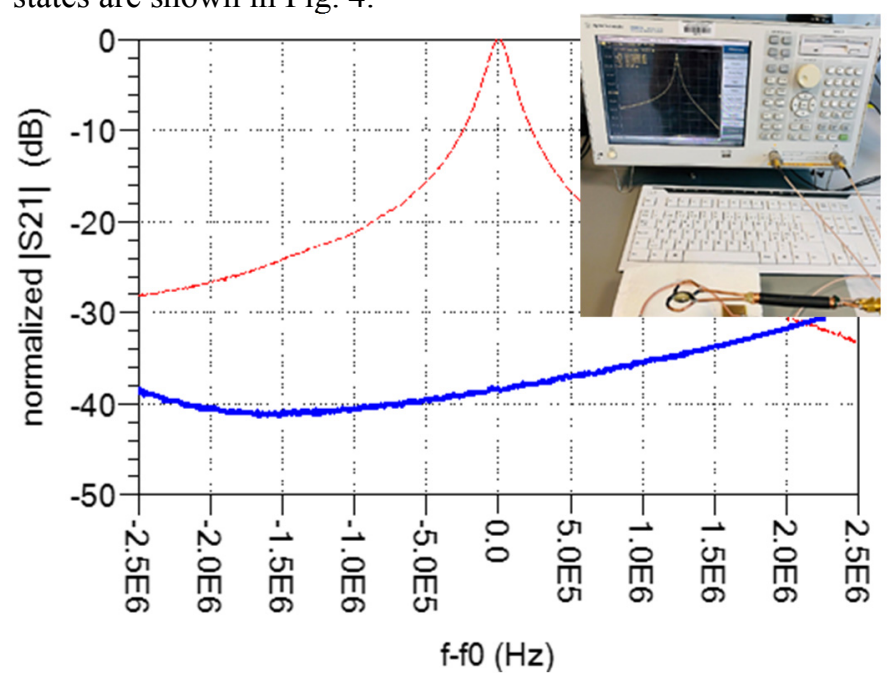

Fig. 4. Decoupling measurements: red - response of the high-impedance resonator; blue - resonator connected to decoupling network.

$38 \mathrm{~dB}$ difference between the states is taken as a measure of decoupling level.

The minimum noise condition is evaluated by observing the noise voltage $V_{\text {out,n }}$ at the output terminals of the LNA in Fig 1. The resulting noise voltage spectrum is shown in Fig. 5.

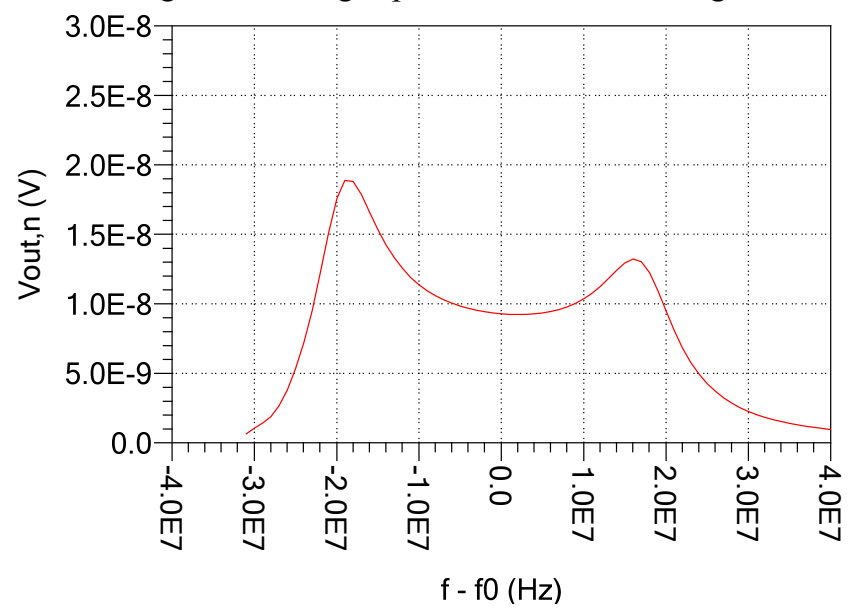

Fig. 5. Noise voltage at the output of the LNA.

As can be observed, there is a local minimum at $f_{0}$. Due to earlier described simplifications in the matching and decoupling circuit, the response is also not symmetric.

\section{IMAGING EXPERIMENTS}

The viability of the proposed method was studied by conducting imaging experiments in a $3 \mathrm{~T}$ MRI scanner.

\section{A. Single Element Test-Efficiency Evaluation}

The imaging properties of the detector were compared to a more traditional low-impedance detector based on the same coaxial cable as well as rigid copper low-impedance detector [7]. All three detectors are based on loops of the same diameter.

Signal-to-noise (SNR) map for the detector using a cylindrical phantom [8] is shown in Fig. 6.

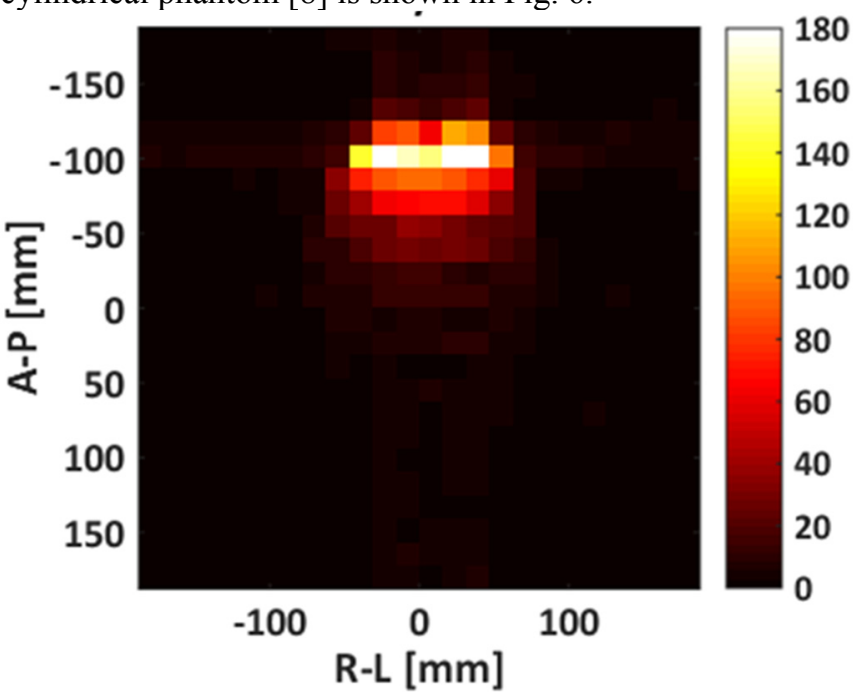

Fig. 6. Imaging. SNR map for high-impedance self-resonant detector. Crosssection through the phantom perpendicular to the plane of the loop: AnteriorPosterior (A-P) and Right-Left (R-L) axis.

As expected, the map follows the intensity of the magnetic field inherent to a loop coil. Similar maps were acquired for the two reference detectors. The resulting SNR profiles are shown in Fig. 7.

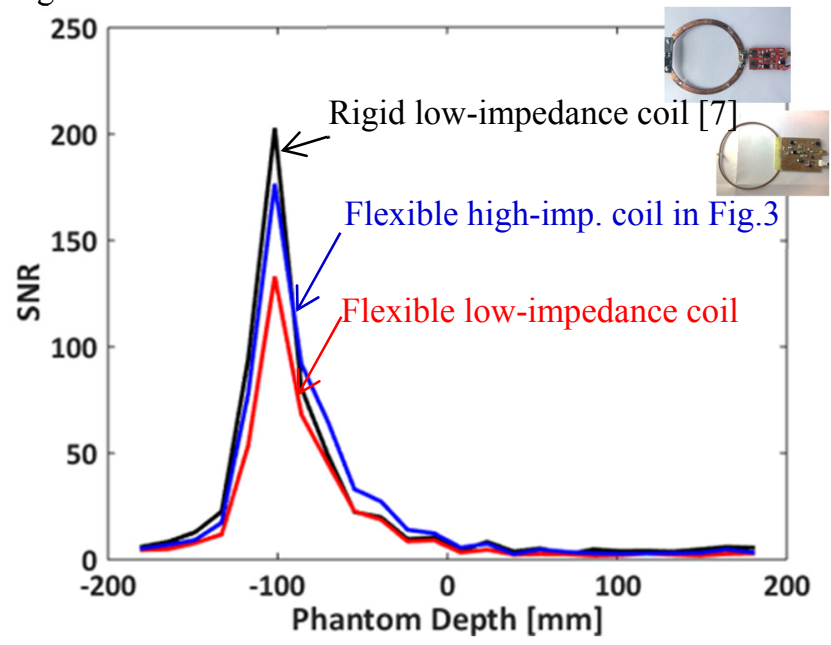

Fig. 7. SNR profile comparison.

The coil is positioned at $-100 \mathrm{~mm}$. One can note signal below $-100 \mathrm{~mm}$ related to a relatively large voxel size required for imaging of low abundance nuclei, such as ${ }^{13} \mathrm{C}$. 


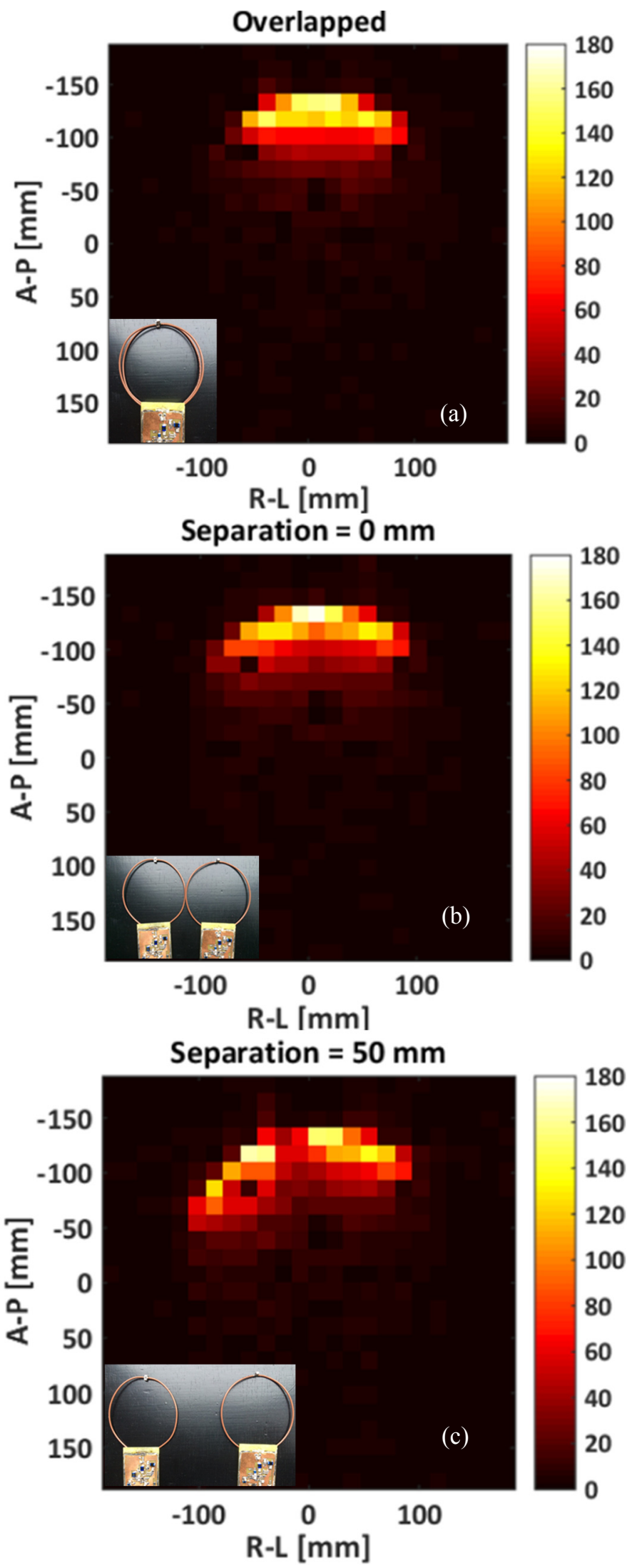

Fig. 8. SNR maps for two element array with different spatial separation. Crosssection through phantom perpendicular to the plane of the coils: AnteriorPosterior (A-P) and Right-Left (R-L) axis.
Obviously, the rigid copper wire has lower conductor loss than the flexible wire which leads to higher SNR. Interesting to note that among flexible realizations, the self-resonant detector exhibits higher SNR.

\section{B. Two Element Array - Decoupling Evaluation}

The results of imaging using two element array placed on a cylindrical phantom [8] for three different coil separations are shown in Fig. 8. In order to evaluate decoupling, the noise correlation between two elements in the array was also calculated and listed in Table 1.

Table 1. Noise correlation

\begin{tabular}{|l|l|l|}
\hline Overlapped & Side to side & Separated $50 \mathrm{~mm}$ \\
\hline 0.206 & 0.213 & 0.181 \\
\hline
\end{tabular}

The noise correlation between the two elements shows small variation for different separations.

\section{CONCLUSION}

A self-resonant high-impedance detector can be tuned by hybrid integration of lumped elements in the coaxial loop structure. Among the variety of different hybrid topologies, using a capacitor in the gap in the outer conductor of the coaxial cable results in the most efficient detector realization. Combining this structure with a noise-matched low-inputimpedance LNA circuit results in lower interaction between elements in the array. Imaging experiments reveal small variation of the noise correlation between channels in the array for different spatial arrangements.

\section{ACKNOWLEDGMENT}

This work was supported in part by the Danish National Research Foundation under Grant DNRF124.

\section{REFERENCES}

[1] P. B. Roemer, W. A. Edelstein, C. E. Hayes, S. P. Souza, O. M. Mueller, "The NMR Phased Array," Magnetic Resonance in Medicine, 16, pp. 192-225, 1990

[2] Zhang B, Sodickson DK, Cloos MA. "A high-impedance detector-array glove for magnetic resonance imaging of the hand," Nature Biomedical Engineering, 2, pp.570-577, 2018.

[3] R. Czerny, L. Nohava, R. Frass-Kriegl, J. Felblinger, J.-C. Ginefri, and E. Laistler, "Flexible multi-turn multi-gap coaxial RF coils: enabling a large range of coil sizes," Proceedings of ISMRM 27th Annual Meeting, Montreal, Canada, 11-16 May, 2019, program \# 1550.

[4] J. Vidkjær, RF Communication Circuits, Chapter 2. [Online]. Available: rftoolbox.dtu.dk.

[5] LNA for MRI, datasheet. [Online]. Available: elcry.com.

[6] W. Wang, V. Zhurbenko, J. D. Sánchez-Heredia, J. H. Ardenkjær-Larsen, "Three-element matching networks for receive-only MRI coil decoupling," accepted to Magnetic Resonance in Medicine, 2020.

[7] J. D. Sanchez-Heredia, A. Ali, W. Wang, R. B. Olin, V. Zhurbenko, Jan H. Ardenkjær-Larsen, "Fixed Geometry 28-Channel Array for Full Head Coverage of Hyperpolarized ${ }^{13} \mathrm{C}$ MRS, " Proceedings of ISMRM 28th Annual Meeting, August 8-14, 2020, program \# 3467.

[8] J. D. Sanchez-Heredia, D. H. Johansen, R. B. Hansen , E. S. Szocska Hansen, C. Laustsen, V. Zhurbenko , and J. H. Ardenkjær-Larsen, "Improved Decoupling for Low Frequency MRI Arrays Using NonConventional Preamplifier Impedance," IEEE Transactions On Biomedical Engineering, Vol. 66, No. 7, pp. 1940-1948, July 2019. 